\title{
HIPERTENSÃO NA DOENÇA RENAL CRÔNICA EM TRATAMENTO CONSERVADOR
}

\author{
HYPERTENSION IN CHRONIC KIDNEY DISEASE UNDER CONSERVATIVE \\ TREATMENT
}

Larissa Parada Leite, ${ }^{1}$ Giovanna Pessanha Cordeiro, ${ }^{1}$ Beatriz Costa Monteiro, ${ }^{1}$ Paulo Gustavo Aguiar de Oliveira, ${ }^{1}$ Pedro Pimenta de Mello Spineti, ${ }^{1}$ Maria Eliane Campos Magalhães, ${ }^{1}$ Erika Maria Gonçalves Campana, ${ }^{1}$ Andréa Araujo Brandão'

\section{RESUMO}

A hipertensão arterial sistêmica (HAS) afeta aproximadamente $30 \%$ da população geral no mundo industrializado e é uma das principais causas de doença renal crônica (DRC), caracterizada por função renal anormal ou danos estruturais renais com mais de 3 meses de duração. A sua presença classifica o paciente hipertenso como de alto risco cardiovascular (CV). O controle pressórico é fundamental para diminuir a sua progressão e reduzir o risco de morbidade e mortalidade CV. A meta pressórica recomendada deve ser menor que $130 \times 80 \mathrm{mmHg}$ para a maioria dos pacientes com DRC. O tratamento não medicamentoso tem como base as mesmas recomendações dos demais pacientes hipertensos. A terapia medicamentosa combinada deve ser iniciada o mais breve possível. Além dos inibidores do sistema renina angiotensina aldosterona, conhecidos por sua eficácia na DRC, os diuréticos e os bloqueadores de canal de cálcio compõem o trio de ouro utilizado no tratamento da HAS, capazes de reduzir o risco cardiovascular e desacelerar a progressão da doença renal. Em conclusão, a DRC é um preditor de risco $\mathrm{CV}$ e a presença concomitante de HAS aumenta expressivamente esse risco. A utilização da estratégia terapêutica adequada para manter os níveis pressóricos dentro das metas é fundamental na atenuação da progressão da doença renal e do risco CV.

Descritores: Hipertensão Arterial Sistêmica; Doença Renal Crônica; Pressão Arterial.

\section{ABSTRACT}

Systemic arterial hypertension (SAH) affects approximately 30\% of the general population in the industrialized world and is a major cause of chronic kidney disease (CKD), characterized by abnormal renal function or structural damage lasting more than 3 months. Its presence classifies the hypertensive as a high cardiovascular (CV) risk patient. Blood pressure (BP) control is essential for slowing the progression and for reducing the risk of CV morbidity and mortality in patients with CKD. BP target $<130 \times 80 \mathrm{mmHg}$ is recommended for most hypertensives with CKD. Non pharmacological treatment is based on the same recommendations for other hypertensive patients. Combination drug therapy should be started as soon as possible. In addition to the renin angiotensin aldosterone system blockers, known for their effectiveness in CKD, diuretics and calcium channel blockers compose the gold trio used in the treatment of SAH, able to reduce cardiovascular risk and to slow the progression of kidney disease. In conclusion, CKD is a predictor of CV risk and the concomitant presence of SAH significantly increases this risk. The use of an appropriate therapeutic strategy to maintain BP control is crucial to mitigate the progression of kidney disease and CV risk.

Keywords: Systemic Arterial Hypertension; Chronic Kidney Disease; Blood Pressure.

\section{EPIDEMIOLOGIA DA ASSOCIAÇÃO ENTRE HIPERTENSÃO ARTERIAL SISTÊMICA E DOENÇA RENAL CRÔNICA}

Hipertensão arterial sistêmica (HAS) afeta aproximadamente $30 \%$ da população geral no mundo industrializado e é uma das principais causas de doença renal crônica (DRC) e da sua progressão para o estágio terminal da doença renal (DRCT), juntamente com o diabetes. A DRC, por si só, também pode agravar as complicações relacionadas à HAS, é um preditor independente de doença cardiovascular (DCV). Nos Estados Unidos, a prevalência de DCV em pacientes com DRC alcança 63\%, já naqueles sem DRC, apenas 5,8\%, ou seja, DRC apresenta prevalência de DCV superior a 9 vezes em relação à da população em geral. O custo geral da DRC é responsável por 1,3\% do orçamento de saúde, dos quais $13 \%$ estão relacionados com o excesso de

1. Faculdade de Ciências Médicas - Universidade do Estado do Rio de Janeiro, RJ, Brasil.

Correspondência: Andréa Araujo Brandão. andreaaraujobrandao@gmail.com

http://dx.doi.org/10.47870/1519-7522/20202704115-21 
infarto agudo do miocárdio e acidentes vasculares cerebrais. Os riscos aumentam à medida em que há redução da taxa de filtração glomerular estimada (TFGe) e aumento dos níveis de albuminúria. A albuminúria sinaliza para a presença de disfunção endotelial, atuando como elo entre as doenças renal e cardiovascular. Um bom controle da pressão arterial (PA) reduz a albuminúria. Nesse contexto, um controle apropriado da PA é importante, especialmente nos pacientes com DRC para evitar complicações da doença renal e a sua progressão. Entretanto, o alvo pressórico a ser mantido em pacientes com DRC ainda é motivo de debate. ${ }^{1-4}$

\section{DOENÇA RENAL CRÔNICA - DEFINIÇÃO, DIAGNÓSTICO E CLASSIFICAÇÃO}

A DRC é definida como anormalidades da função renal ou danos estruturais renais presentes por um período maior do que 3 meses, com implicações negativas na saúde. Isso inclui todos aqueles pacientes com marcadores de lesão renal (albuminúria, razão albumina: creatinina (ACR) maior que $3 \mathrm{mg} / \mathrm{mmol}$, sedimentos urinários anormais, distúrbios eletrolíticos e outras anormalidades relacionadas à lesão tubular, anormalidades detectáveis pela histologia, anormalidades estruturais detectáveis por imagem e história de transplante renal) e aqueles com taxa de filtração glomerular (TFGe) menor que $60 \mathrm{ml} / \mathrm{min} / 1.73 \mathrm{~m}^{2}$ em pelo menos duas ocasiões separadas por um período de 90 dias (com ou sem marcadores de lesão renal). ${ }^{5}$

O diagnóstico da DRC pode ser realizado a partir de testes simples (TFGe e ACR), mas, por vezes, esta condição clínica só é reconhecida ou diagnosticada em estágios avançados, por ser usualmente assintomática e/ou não ter sintomas específicos. O risco de desenvolver DRC aumenta com a idade, e ela frequentemente, coexiste com outras condições clínicas, como doença cardiovascular e diabetes. DRC pode progredir para DRCT em pequena, mas significante porcentagem de pessoas. ${ }^{5}$

DRC é classificada de acordo com a TFGe e ACR, pois indicam risco de eventos adversos e progressão da DRC, e quando usados de forma conjunta, melhoram a acurácia e a estratificação de risco. Em 2013, foi publicada a diretriz de DRC do Kidney Disease: Improving Global Outcomes (KDICO) definindo a classificação mais atualizada da DRC (Tabela1). ${ }^{5}$

\section{DOENÇA RENAL CRÔNICA E O RISCO CARDIOVASCULAR}

A presença de DRC classifica automaticamente o paciente hipertenso como de alto risco CV. A presença de microalbuminúria aumenta em até quatro vezes o risco CV. Além disso, pode-se observar uma relação inversamente proporcional entre TFGe e risco CV. Pacientes em estágio 3 (TFGe 30-59 $\mathrm{ml} / \mathrm{min} / 1,73 \mathrm{~m}^{2}$ ) elevam o risco de duas a quatro vezes; no estágio 4 (TFGe $15-29 \mathrm{ml} / \mathrm{min} / 1,73 \mathrm{~m}^{2}$ ) o risco passa de quatro a dez vezes e no estágio 5 (TFGe $<15 \mathrm{ml} / \mathrm{min} / 1,73 \mathrm{~m}^{2}$ ) observa-se um aumento de dez a cinquenta vezes. Liu M e colaboradores, em sua meta-análise, também apontaram um aumento da mortalidade em paciente com DRC, sendo essa taxa de $40 \%$ na população geral (sem DRC), >50\% em paciente com DRC, e em pacientes com DRCT pode-se observar uma elevação de até 15 vezes, se comparada à população sem DRC. Essas proporções se mantêm quando observada a população chinesa, na qual as DCV correspondem a cerca de $44,2 \%$ - 51,0\% da mortalidade geral entre pacientes em diálise. Além disso, em pacientes com DRCT que iniciaram a diálise, é possível observar que aproximadamente $73,4 \%$ apresentam hipertrofia do ventrículo esquerdo (HVE), 35,8\% possuem dilatação do VE e 14,8\% têm disfunção sistólica do

Tabela 1. Classificação da doença renal crônica usando as categorias de TFGe e ACR.

\begin{tabular}{|c|c|c|c|c|c|c|}
\hline \multirow{3}{*}{\multicolumn{3}{|c|}{$\begin{array}{c}\text { Categorias GFR e ACR e risco de resultados } \\
\text { adversos }\end{array}$}} & \multicolumn{3}{|c|}{ Categorias ACR (mg/mol), descrição e nível } & \multirow{9}{*}{ 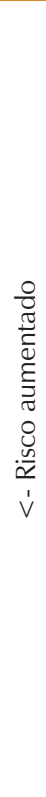 } \\
\hline & & & $\begin{array}{l}<3 \\
\text { Normal/levemente } \\
\text { aumentada }\end{array}$ & $\begin{array}{l}\text { 3-30 } \\
\text { Moderadamente } \\
\text { aumentada }\end{array}$ & $\begin{array}{l}>30 \\
\text { Gravemente } \\
\text { aumentada }\end{array}$ & \\
\hline & & & A1 & A2 & A3 & \\
\hline \multirow{6}{*}{$\begin{array}{l}\text { Categorias GFR } \\
\left(\mathrm{ml} / \mathrm{mim} / 1.73 \mathrm{~m}^{2}\right), \\
\text { descrição e nível }\end{array}$} & $\begin{array}{l}\geq 30 \\
\text { Normal/alto }\end{array}$ & G1 & \multirow[b]{2}{*}{$\begin{array}{l}\text { Sem DRC na ausência } \\
\text { de marcadores de } \\
\text { Lesão renal }\end{array}$} & & & \\
\hline & $\begin{array}{l}60-89 \\
\text { Redução leve em } \\
\text { relação ao nível } \\
\text { normal para um } \\
\text { adulto jovem }\end{array}$ & G2 & & & & \\
\hline & $\begin{array}{l}45-59 \\
\text { Redução leve- } \\
\text { moderada }\end{array}$ & G3a & & & & \\
\hline & $\begin{array}{l}\text { 30-44 } \\
\text { Redução } \\
\text { moderada-grave }\end{array}$ & $\mathrm{G} 3 \mathrm{~b}$ & & & & \\
\hline & $\begin{array}{l}\text { 15-29 } \\
\text { Redução grave }\end{array}$ & G4 & & & & \\
\hline & $\begin{array}{l}<15 \\
\text { Falência renal }\end{array}$ & G5 & & & & \\
\hline
\end{tabular}

Risco aumentado ->

Abreviações: ACR, razão alburnina:creatinina; DRC, doença renal crônica; GFR, taxa de filtração glomerular. Adaptada de Forbes $\mathrm{A}$, Gallagher H. Chronic kidney disease in adults: assessment and managernenit [published online ahead of print, 2020 Mar 121. Clin Med (Lond). 2020;20(2):128- 132 doi:10.7861/clinrned.cg.202. O aumento do risco de resultados adversos está representado na tabela pela progressão de cores, do laranja mais claro (representando menor risco) para o laranja mais escuro (representando maior risco). 
VE. Szu-Chia Chen e colaboradores também apontaram que pacientes com DRC apresentavam uma maior prevalência de aumento da massa ventricular esquerda (MVE) quando comparado com pacientes que apresentam a função renal normal $(52,6 \% \text { vs. } 30,5 \%,)^{2,6,7}$

\section{METAS DE PRESSÃO ARTERIAL}

O adequado controle pressórico é fundamental para diminuir a progressão da lesão renal e reduzir o risco de morbidade e mortalidade CV no portador de DRC. Entretanto, a meta pressórica ideal neste grupo de pacientes ainda é motivo de controvérsia na literatura. Alguns pesquisadores defendem que o ideal seria a utilização de metas mais rigorosas $(<120 \mathrm{mmHg})$. Um estudo realizado durante um ano com 9.316 participantes, sendo 2646 com DRC, demonstrou que pacientes com metas mais rigorosas, quando comparados com pacientes com a meta padrão (<140mmHg), apresentaram uma menor redução de TFGe $(0,23 \%$ contra 0,26\%); menor ocorrência de eventos cardiovasculares $(0,33 \%$ contra $0,36 \%)$; menor necessidade de diálise a longo prazo $(0,14 \%$ contra $0,24 \%)$ e uma redução de $9 \%$ no risco da progressão da albuminúria. Kim Chang Seong e colaboradores, em sua meta-análise, também relataram redução de $25 \%$ de risco de doenças cardiovasculares e uma redução de $27 \%$ na morte por qualquer causa em pacientes que seguem uma meta <120mmHg em comparação com pacientes com metas $<140 \mathrm{mmHg} .{ }^{8,9}$

Em contraposição, Kovesdy C, juntamente com seus colaboradores, realizaram um estudo de observação de PA de veteranos dos EUA, o qual evidenciou que PAS $<120 \mathrm{mmHg}$ estava associada ao aumento da mortalidade, independentemente do valor de PAD. Em outro estudo observacional do mesmo grupo sobre o controle de pressão arterial estrito versus convencional em pacientes com DRC, os autores ratificaram essa teoria, relatando a existência de uma curva J da PA em pacientes com DRC tratados com metas muito reduzidas. Para tal, utilizaram dados de 77.765 pacientes com DRC, cerca de 5760 foram tratados com meta $<120 \mathrm{mmHg}$ e 72.005 com PA entre $120-139 \mathrm{mmHg}$. A taxa de mortalidade, em 6 anos, em pacientes com a meta mais rígida foi de 80,9/1000 pacientes-ano, enquanto para pacientes com a meta entre $120-139 \mathrm{mmHg}$ a mortalidade foi $41,8 / 1000$ pacientes-ano. ${ }^{10,11}$

Desta forma, a diretriz europeia de hipertensão arterial de 2018 recomenda uma meta de PA entre os pacientes portadores de DRC entre $<140-130 /<80-70 \mathrm{mmHg}$. Entretanto, a $7^{\mathrm{a}}$ Diretriz Brasileira de Hipertensão, define metas mais agressivas para estes pacientes, por considerá-los uma população de alto risco, como podemos verificar na Tabela $2 .^{14,15}$

Tabela 2. Metas pressóricas para pacientes em tratamento conservador, de acordo com a etiologia da doença renal e com a excreção urinária de albumina*.

\begin{tabular}{l|c|c} 
& Albuminúria $<30 \mathrm{mg} / 24 h$ & Albuminúria $>30 \mathrm{mg} / 24 h$ \\
$\begin{array}{l}\text { DRC não } \\
\text { diabética }\end{array}$ & $<140 / 90 \mathrm{mmHg}$ & $<130 / 80 \mathrm{mmHg}$ \\
\hline $\begin{array}{l}\text { DRC } \\
\text { diabética }\end{array}$ & $<130 / 80 \mathrm{mmHg}$ & $<130 / 80 \mathrm{mmHg}$ \\
\hline
\end{tabular}

Tabela adaptada da $7^{a}$ Diretriz Brasileira de Hipertensão1. *Metas < 120/70 mmHg não devem ser utilizadas em pacientes com doença coronariana associada.

\section{TRATAMENTO NÃO MEDICAMENTOSO DA HIPERTENSÃO ARTERIAL}

O tratamento não medicamentoso (TNM) da hipertensão arterial nos pacientes com DRC tem como base o mesmo TNM dos demais pacientes hipertensos. De acordo com a 7ạ Diretriz Brasileira de Hipertensão Arterial o controle do peso, medidas nutricionais, atividades físicas, entre outras mudanças no estilo de vida (MEV), devem ser estimuladas concomitante ao tratamento medicamentoso. ${ }^{15}$

Dentre as várias abordagens dietéticas, a mais recomendada com efeito hipotensor é a dieta DASH. Ela é baseada na elevada ingestão de frutas, vegetais e cálcio e reduzido consumo de gordura saturada, resultando em diminuição da pressão, mesmo quando não há perda de peso e/ou restrição salina. No Brasil, essa dieta é recomendada, porém necessita de melhorias para garantir a adesão do paciente. Algumas estratégias podem ser úteis, como o tratamento flexível, simplificação das orientações, mudanças graduais, abordagem multiprofissional e utilização de ferramentas digitais. ${ }^{16,17}$

A dieta com alto teor de sódio diminui a autorregulação renal, expondo o glomérulo a pressões mais altas de filtração, o que leva à nefroesclerose e perda de néfrons, por isso, é recomendado a restrição salina na dieta. Com a ingestão de aproximadamente $3 \mathrm{~g} /$ dia de sal é possível diminuir a PA sistólica em mais de $10 \mathrm{mmHg}$, e para alvos de $6 \mathrm{~g} /$ dia de sal pode-se observar redução de cerca de $25 \%$ na proteinúria. Em hipertensão arterial resistente, recomenda-se até $2 \mathrm{~g}$ de sódio/dia, o que equivale a $5 \mathrm{~g}$ de sal. ${ }^{15,18}$

No que diz respeito à atividade física, ela possui impacto na pressão arterial com redução de até 8,3/5,2 mmHg, principalmente no exercício aeróbico. Diante disso, recomenda-se o mínimo de $30 \mathrm{~min} /$ dia de atividade física moderada, de forma contínua ( 1 x 30 min) ou acumulada $(2 \times 15$ min ou 3 x 10 min) em 5 a 7 dias da semana. No entanto, indivíduos com pressões arteriais mais elevadas e/ou com três ou mais fatores de risco devem antes realizar avaliação cardiológica previamente ao início da prática de exercícios físicos. ${ }^{15,19}$

As MEV podem impactar positivamente na redução da massa corpórea. Existe uma estreita relação entre o peso corporal e a pressão arterial. Há estimativa de que a cada 5\% de ganho de peso gere um aumento de $20 \%$ a $30 \%$ nas chances de desenvolver a doença. Por isso, é recomendado manter o índice de massa corporal (IMC) $<25 \mathrm{~kg} / \mathrm{m}^{2}$ em indivíduos até 65 anos e IMC $<27 \mathrm{~kg} / \mathrm{m}^{2}$ após 65 anos. Além disso, o aumento da gordura visceral também representa um importante fator de risco, sendo, portanto, recomendado manter a circunferência abdominal $<80 \mathrm{~cm}$ nas mulheres e $<94 \mathrm{~cm}$ nos homens. ${ }^{15,20,21}$

\section{TRATAMENTO MEDICAMENTOSO DA HIPERTENSÃO ARTERIAL}

A terapia medicamentosa, em combinação com o TNM, é estratégia obrigatória para se atingir as metas de PA recomendadas na DRC. (Figura 1) Algumas classes de medicamentos anti-hipertensivos, de forma independente da PA, são capazes de reduzir o risco cardiovascular e desacelerar a progressão da doença renal. ${ }^{14,15,18}$

É importante ressaltar que, no início do tratamento antihipertensivo pode haver uma pequena queda da taxa de 




DRC: Doença Renal Crônica; MEV: Mudança no Estilo de Vida; IECA: Inibidores da Enzima Conversora de Angiotensina; BRA: Bloqueadores do Receptor de Angiotensina; BCC: Bloqueadores do Canal de Cálcio.

Figura 1. Esquema terapêutico na Doença Renal Crônica.

filtração glomerular. Esse evento, observado pelo aumento da creatinina sérica, decorre da diminuição da perfusão renal pela redução da pressão arterial, que leva a uma redução da pressão intraglomerular. Entretanto, como esse declínio tende a se estabilizar após as primeiras semanas de uso dos medicamentos, recomenda-se a continuidade da terapia já que esta cursa com renoproteção em longo prazo. Caso essa redução persista por mais tempo ou o aumento inicial da creatinina sérica seja superior a 30\%, é necessário interromper a terapia e avaliar a presença de outros fatores associados, como a doença renovascular. Desta forma, deve-se realizar um monitoramento da microalbuminúria, eletrólitos sanguíneos e da TFGe durante o uso dos medicamentos para se acompanhar cuidadosamente os possíveis efeitos adversos da terapia anti-hipertensiva. ${ }^{14,18,22-24}$

A monoterapia resulta em uma taxa limitada de alcance das metas de PA, apenas 30\%. Desta forma, a $7^{\text {a }}$ Diretriz Brasileira de Hipertensão, recomenda para pacientes com alto risco CV o uso de terapia combinada desde o início do tratamento para atingir as metas recomendadas. Pacientes com DRC devem fazer uso de terapia combinada o mais breve possível. ${ }^{15}$

Para a escolha da classe de fármaco ideal, é necessário considerar dois aspectos importantes na DRC: se é de origem diabética e quanto à presença de proteinúria ( $\geq 300$ mg em urina de 24 h ou relação albumina/creatinina urinária $\geq 0,3$ $\mathrm{mg} / \mathrm{mg}$ ). Na DRC não diabética pode ser utilizada qualquer classe de fármaco para atingir a meta, pois a redução da PA é a estratégia mais eficaz para retardar a perda da função renal; entretanto, caso haja albuminúria, os fármacos bloqueadores do sistema renina angiotensina-angiotensinogênio (SRAA) são os de escolha por seus efeitos renoprotetores, e deve-se atingir uma meta agressiva de redução pressórica, $<130 \times 80$ mmHg. ${ }^{15,25}$

Na DRC diabética, os bloqueadores do SRAA são obrigatórios por apresentarem eficácia tanto no controle da PA quanto na redução da albuminúria na DRC. O estudo ADVANCE demonstrou que o uso do iECA perindopril associado à indapamida apresentou redução significativa de eventos renais, como da piora da nefropatia, de modo que é capaz de reduzir o risco de morte prematura em pacientes com DRC diabética. (Figura 2)
De acordo com a meta-análise realizada por Shen e colaboradores, sugere-se que os iECA são altamente eficazes na proteção ao desenvolvimento de DRCT em pacientes com DRC diabética, reduzindo a mortalidade em todas as causas (RR: 0,90, IC 95\%: 0,86-0,95), mesmo quando utilizados isoladamente em pacientes diabéticos. ${ }^{14,15,24-27}$

Vejakama e colaboradores avaliaram 15.032 pacientes com DRC diabética e 17.074 com DRC não diabética randomizados para o uso de bloqueadores do SRAA por tempo menor e maior que um ano, quanto à progressão para DRCT e morte antes do início de DRCT. Os pacientes com DRC diabética tiveram risco de progredir para DRCT de 19,0\% quando fizeram uso dos fármacos por um período inferior a um ano, e de 12,9\% quando fizeram uso dos fármacos por um período superior a um ano, demonstrando que o uso de fármacos por um período superior a um ano foi capaz de evitar tanto a progressão da doença renal, quanto a morte antes do início de DRCT nesse grupo. Pela proteção que conferem ao rim, os bloqueadores do SRAA são imprescindíveis no tratamento desse grupo de pacientes..$^{15,28}$

Além dos inibidores do SRAA, os diuréticos e os bloqueadores de canal de cálcio (BCC) compõem o trio de ouro no tratamento da HAS. Adicionar um BCC diidropiridínico a um bloqueador do SRAA melhora o controle da PA e tem efeito positivo na redução da proteinúria. Com isso, a combinação de bloqueadores do SRAA e BCC é preferível à combinação com diuréticos, já que este último, pode se associar a aumento do risco de novos casos de DM tipo 2, com consequente agravo adicional da função renal destes pacientes. Além disso, o estudo ACCOMPLISH, que comparou as combinações terapêuticas do iECA benazepril com amlodipina e com hidroclorotiazida, demonstrou eficácia maior da primeira em reduzir mortalidade por $\mathrm{DCV}(\mathrm{RR}=0,80 ; \mathrm{P}<0,001)$ e a progressão da $D R C(R R=0,79 ; P=0,002)$ (Figura 3). $A$ associação entre iECA e BRA não é recomendada por não demonstrar benefícios adicionais e aumentar a ocorrência de efeitos adversos, como lesão renal aguda e hipercalemia, mesmo na DRC por doença renal policística. ${ }^{15,18,25,29}$

A hipervolemia é um fator importante na fisiopatologia da 


\begin{tabular}{|c|c|c|c|c|c|}
\hline & \multicolumn{2}{|c|}{$\begin{array}{l}\text { Número (\%) de pacientes } \\
\text { com eventos }\end{array}$} & \multirow{2}{*}{$\begin{array}{l}\text { Favorece } \\
\text { perindopril- } \\
\text { indapamida }\end{array}$} & \multirow[t]{2}{*}{$\begin{array}{l}\text { Favorece } \\
\text { placebo }\end{array}$} & \multirow{2}{*}{$\begin{array}{l}\text { Redução do } \\
\text { risco relativo } \\
\text { (IC 95\%) }\end{array}$} \\
\hline & $\begin{array}{l}\text { Perindopril- } \\
\text { indapamida } \\
(\mathrm{n}=5569)\end{array}$ & $\begin{array}{l}\text { Placebo } \\
(n=5571)\end{array}$ & & & \\
\hline Combinação Macro + Micro & $861(15 \cdot 5 \%)$ & $938(16 \cdot 8 \%)$ & & & $9 \%(0$ a 17$)$ \\
\hline Macrovascular & $480(8.6 \%)$ & $520(9 \cdot 3 \%)$ & & - & $8 \%(-4$ a 19$)$ \\
\hline Microvascular & $439(7 \cdot 9 \%)$ & $477(8.6 \%)$ & & - & $9 \%(-4$ a 20$)$ \\
\hline Todas as mortes & $408(7 \cdot 3 \%)$ & $471(8 \cdot 5 \%)$ & & & $14 \%(2$ a 25$)$ \\
\hline Morte cardiovascular & $211(3-8 \%)$ & $257(4 \cdot 6 \%)$ & & & $18 \%(2$ a 32$)$ \\
\hline Morte por doença não cardiovascular & $197(3-5 \%)$ & $212(3 \cdot 8 \%)$ & & & $8 \%(-12$ a 24$)$ \\
\hline Eventos coronarianos totais & $468(8 \cdot 4 \%)$ & $535(9 \cdot 6 \%)$ & & & $14 \%(2$ a 24$)$ \\
\hline Eventos coronarianos maiores & $265(4 \cdot 8 \%)$ & $294(5 \cdot 3 \%)$ & & & $11 \%(-6$ a 24$)$ \\
\hline Outros eventos coronarianos* & $283(5 \cdot 1 \%)$ & $324(5 \cdot 8 \%)$ & & & $14 \%(-1$ a 27$)$ \\
\hline Total de eventos cerebrovasculares & $286(5 \cdot 1 \%)$ & $303(5 \cdot 4 \%)$ & & - & $6 \%(-10$ a 20$)$ \\
\hline Eventos cerebrovasculares maiores & $215(3-9 \%)$ & $218(3 \cdot 9 \%)$ & & & $2 \%(-18$ a 19$)$ \\
\hline Outros eventos cerebrovasculares \# & $79(1-4 \%)$ & $99(1-8 \%)$ & & & $21 \%(-6$ a 41$)$ \\
\hline Eventos renais totais & $1243(22 \cdot 3 \%)$ & $1500(26 \cdot 9 \%)$ & & & $21 \%(15$ a 27$)$ \\
\hline Nefropatia nova ou piora & $181(3 \cdot 3 \%)$ & $216(3 \cdot 9 \%)$ & & & $18 \%(-1$ a 32$)$ \\
\hline Microalbuminúria nova & $1094(19 \cdot 6 \%)$ & $1317(23 \cdot 6 \%)$ & $\vdash$ & & $21 \%(14$ a 27$)$ \\
\hline Eventos oculates totais & $2531(45 \cdot 4 \%)$ & $2611(46 \cdot 9 \%)$ & & & $5 \%(-1$ a 10$)$ \\
\hline Retinopatia nova ou piora & $289(5 \cdot 2 \%)$ & $286(5 \cdot 1 \%)$ & & & $-1 \%(-18$ a 15$)$ \\
\hline Deterioração Visual & 2446 (43-9\%) & $2514(45-1 \%)$ & & & $5 \%(-1$ a 10$)$ \\
\hline & & 0.5 & 1 . & 0 & $2 \cdot 0$ \\
\hline \multicolumn{3}{|l|}{ Adaptada do estudo Advance. ${ }^{26}$} & \multicolumn{2}{|c|}{ Razão de risco } & \\
\hline
\end{tabular}

Figura 2. Estudo ADVANCE. Efeito do tratamento com perindopril-indapamida nas mortes, eventos coronarianos, cerebrovasculares, renais e oculares em pacientes diabéticos.

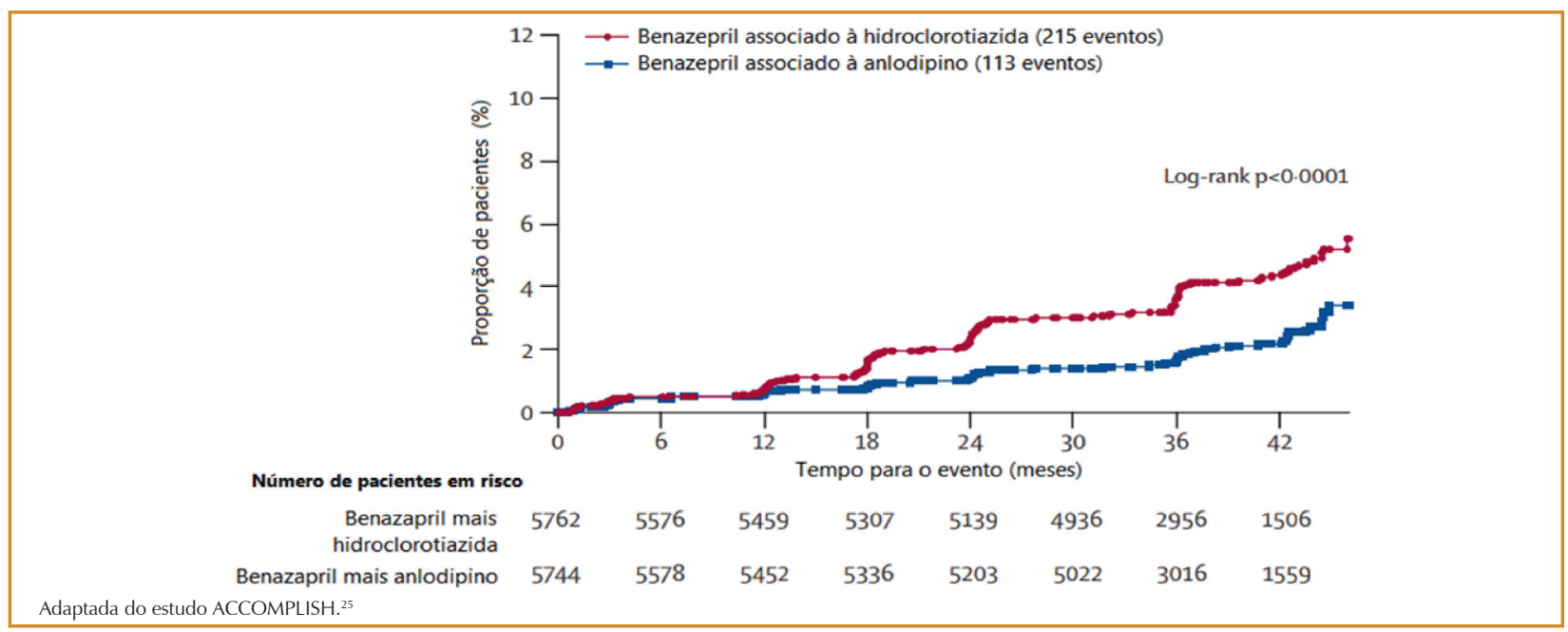

Figura 3. Estudo ACCOMPLISH. Progressão da doença renal crônica em hipertensos de alto risco cardiovascular. Análise da população por intenção de tratar. A progressão da doença renal crônica foi definida como o dobro da concentração de creatinina sérica, taxa de filtração glomerular estimada inferior a $15 \mathrm{~mL} / \mathrm{min} / 1,73 \mathrm{~m} 2$ ou necessidade de diálise. 
HAS na DRC, e a sobrecarga de volume subclínica pode afetar até $50 \%$ desses pacientes. Para o controle da PA nos estágios iniciais da DRC (1, 2 e 3) os tiazídicos (hidroclorotiazida, clortalidona) são os diuréticos de escolha, enquanto que em situações de edema e nos estágios mais avançados (4 e 5) os diuréticos de alça são preferidos (quando TFGe $\leq 30 \mathrm{ml} / \mathrm{min} / 1,73 \mathrm{~m}^{2} \mathrm{ou}$ creatinina $>2,0 \mathrm{mg} / \mathrm{dl}$ ). O uso de diuréticos durante a terapia dialítica é recomendado se houver presença de função renal residual, por reduzir mortalidade e controlar a PA. ${ }^{15,18}$

Para pacientes em diálise, apesar de todos os medicamentos citados poderem ser utilizados, a escolha da terapia anti-hipertensiva deve ser individualizada de acordo com as características clínicas de cada paciente e com as características farmacocinéticas dos medicamentos escolhidos, assim como seus efeitos adversos. ${ }^{15,18,30,31}$

\section{DENERVAÇÃO RENAL}

A denervação simpática renal (DSR) consiste na ablação mais comumente por radiofrequência, mas também por ultrassom, dos nervos que inervam a artéria renal com intuito de reduzir a PA. Um estudo com pacientes com DRC mostrou reduções notáveis na pressão arterial (> 30 mmHg) após DSR na doença mais avançada. Outros estudos mostraram ainda reduções da albuminúria e alentecimento na progressão da DRC. Em decorrência dos resultados controversos na utilização da DSR, o procedimento não está autorizado na prática clínica, sendo necessárias pesquisas mais robustas para sua aplicação no tratamento da hipertensão. ${ }^{32,33}$

\section{CONCLUSÃO}

A doença renal crônica é uma condição associada a alto risco $\mathrm{CV}$, e a presença concomitante de HAS aumenta expressivamente esse risco, pois acelera a progressão da lesão renal para doença renal crônica terminal, assim como eleva também a mortalidade cardiovascular. A utilização da estratégia terapêutica adequada para manter os níveis pressóricos dentro das metas estabelecidas para esta população é fundamental na atenuação da progressão da doença renal e na redução de evolução para doença renal crônica terminal. O controle pressórico deve ser realizado com o uso de terapia combinada e, dentro dessa associação, o bloqueio do SRAA é fundamental. Entretanto, os valores referentes às metas de PA ideais nestes pacientes permanecem como ponto de discussão importante para os médicos.

\section{REFERÊNCIAS}

1. Stompór T, Perkowska-Ptasińska A. Hypertensive kidney disease: a true epidemic or rare disease? Pol Arch Intern Med. 2020 Feb 27;130(2):130139. doi: 10.20452/pamw.15150. Epub 2020 Jan 22.

2. Liu M, Li XC, Lu L, et al. Cardiovascular disease and its relationship with chronic kidney disease. Eur Rev Med Pharmacol Sci. 2014;18(19):2918-26.

3. Major RW, Cheng MRI, Grant RA, et al. Cardiovascular disease risk factors in chronic kidney disease: A systematic review and meta-analysis. PLoS One. 2018;13(3):e0192895. doi:10.1371/journal.pone.0192895

4. Cha RH, Lee H, Lee JP, Kim YS, Kim SG. The influence of blood pressure patterns on renal outcomes in patients with chronic kidney disease: The long-term follow up result of the APrODiTe-2 study. Medicine (Baltimore). 2020;99(8):e19209. doi:10.1097/MD.0000000000019209

5. Forbes A, Gallagher H. Chronic kidney disease in adults: assessment and management [published online ahead of print, 2020 Mar 12]. Clin Med (Lond). 2020;20(2):128-32. doi:10.7861/clinmed.cg.20.2

6. Perk J, De Backer G, Gohlke H, et al. European Guidelines on cardiovascular disease prevention in clinical practice (version 2012). The Fifth Joint Task Force of the European Society of Cardiology and Other Societies on Cardiovascular Disease Prevention in Clinical Practice (constituted by representatives of nine societies and by invited experts) Eur Heart J. 2012 Jul;33(13):1635-701. doi: 10.1093/eurheartj/ehs092. Epub 2012 May 3. Erratum in: Eur Heart J. 2012 Sep;33(17):2126.

7. Chen SC, Huang JC, Su HM, et al. Prognostic Cardiovascular Markers in Chronic Kidney Disease. Kidney Blood Press Res. 2018;43(4):1388-1407. doi:10.1159/000492953

8. SPRINT, Wright JT Jr, Williamson JD, et al. A Randomized Trial of Intensive versus Standard Blood-Pressure Control [correção publicada aparece em N Engl J Med. 21 de dezembro de 2017; 377 (25): 2506]. N Engl J Med. 2015; 373(22):2103-16. doi: 10.1056/ NEJMoa1511939

9. Kim CS, Choi HS, Bae EH, Kim SW, Ma SK. Optimal blood pressure target and measurement in patients with chronic kidney disease. Korean J Intern Med. 2019;34 (6):1181-7. doi: 10.3904 / kjim.2019.164

10. Kovesdy CP, Bleyer AJ, Molnar MZ, et al. Blood pressure and mortality in U.S. veterans with chronic kidney disease: a cohort study.. Ann Intern Med. 2013;159(4):233-42. doi: 10.7326 / 0003-4819-159-4201308200-00004

11. Kovesdy CP, Lu JL, Molnar MZ, et al. Observational modeling of strict vs conventional blood pressure control in patients with chronic kidney disease [published correction appears in JAMA Intern Med. 2014 Oct;174(10):1709].JAMA Intern Med. 2014;174(9):1442-9. doi:10.1001/ jamainternmed.2014.3279

12. Viazzi F, Leoncini G, Grassi G, Pontremoli R. Antihypertensive treatment and renal protection: Is there a J-curve relationship?. J Clin Hypertens (Greenwich). 2018;20(11):1560-74. doi:10.1111/jch.13396

13. Kollias A, Kyriakoulis KG, Stergiou GS. Blood pressure target for hypertension in chronic kidney disease: One size does not fit all. J Clin Hypertens (Greenwich). 2020;22(5):929-32. doi:10.1111/jch.13861

14. Williams B, Mancia G, Spiering W, et al.2018 ESC/ESH Guidelines for the management of arterial hypertension: The Task Force for the management of arterial hypertension of the European Society of Cardiology (ESC) and the European Society of Hypertension (ESH). European Heart Journal. 2018;39(33):3021-104. doi: 10.1093/eurheartj/ehy339

15. Malachias MVB, Souza WKSB, Plavnik FL, et al. $7 \stackrel{\text { a }}{ }$ Diretriz Brasileira de Hipertensão Arterial. Arq Bras Cardiol. 2016; 107(3):supl 3.

16. Sacks FM, Svetkey LP, Vollmer WM, et al. ; DASH-Sodium Collaborative Research Group. Effects on blood pressure of reduced dietary sodium and the Dietary Approaches to Stop Hypertension (DASH) diet. DASH-Sodium Collaborative Research Group. N Engl J Med. 2001;344(1):3-10. doi: 10.1056/NEJM200101043440101

17. Bricarello LP, Retondario A, Poltroneri F, Souza AM, Vasconcelos FAG. A dietary approach to control hypertension: reflections on adherence to and possible impacts on public health/Abordagem dietetica para controle da hipertensao: reflexoes sobre adesao e possiveis impactos para a saude coletiva. Ciência \& Saúde Coletiva. 2020 25(4):1421-33.

18. Pugh D, Gallacher PJ,Dhaun N. Management of hypertension in chronic kidney disease. Drugs. 2019;79(4):365-79.

19. Cornelissen VA, Smart NA. Exercise training for blood pressure: a systematic review and meta-analysis. J Am Heart Assoc. 2013; 2(1):e004473. doi: 10.1161/JAHA.112.004473.

20. DeMarco VG, Aroor AR, Sowers JR. The pathophysiology of hypertension in patients with obesity. Nat Rev Endocrinol. 2014; 10(6): 364-76. doi: $10.1038 /$ nrendo.2014.44 
21. Vaneckova I,Maletínská L, Behuliak M, et al. Obesity-related hypertension: possible pathophysiological mechanisms. J Endocrinol. 2014;223(3):R63-78.

22. Collard D, Brouwer TF, Olde Engberink RHG, Zwinderman AH, Vogt L, van den Born BH. Initial Estimated Glomerular Filtration Rate Decline and Long-Term Renal Function During Intensive Antihypertensive Therapy: A Post Hoc Analysis of the SPRINT and ACCORD-BP Randomized Controlled Trials.Hypertension.2020;75(5):1205-212. doi:10.1161/ HYPERTENSIONAHA.119.14659

23. Whelton PK, Carey RM, Aronow WS, et al. 2017 ACC/AHA/AAPA/ABC/ ACPM/AGS/APhA/ASH/ASPC/NMA/PCNA Guideline for the Prevention, Detection, Evaluation, and Management of High Blood Pressure in Adults. Hypertension 2018;71:e13-e115. doi: 10.1161/HYP.0000000000000065

24. Unger T, Borghi C, Charchar F, et al. 2020 International Society of Hypertension Global Hypertension Practice Guidelines. Hypertension. 2020;75:1-24.

25. Rabi DM, McBrien KA, Sapir-Pichhadze R. Hypertension Canada's 2020 Comprehensive Guidelines for the Prevention, Diagnosis, Risk Assessment, and Treatment of Hypertension in Adults and Children. Can J Cardiol. 2020;36:596:624. doi: 10.1016/j.cjca.2020.02.086

26. Patel A; ADVANCE Collaborative Group, MacMahon S, et al. Effects of a fixed combination of perindopril and indapamide on macrovascular and microvascular outcomes in patients with type 2 diabetes mellitus (the ADVANCE trial): a randomised controlled trial. Lancet. 2007;370(9590):829-840. doi:10.1016/S0140-6736(07)61303-8

27. Shen J, Huang YM, Song XN, et al. Protection against death and renal failure by renin-angiotensin system blockers in patients with diabetes and kidney disease. J Renin Angiotensin Aldosterone Syst. 2016;17(3):1470320316656481. doi:10.1177/1470320316656481

28. Vejakama P, Ingsathit A, McKay GJ, et al. Treatment effects of reninangiotensin aldosterone system blockade on kidney failure and mortality in chronic kidney disease patients. BMC Nephrol. 2017;18(1):342. doi:10.1186/s12882-017-0753-9

29. Bakris GL, Sarafidis PA, Weir MR, et al. Renal outcomes with different fixed-dose combination therapies in patients with hypertension at high risk for cardiovascular events (ACCOMPLISH): a prespecified secondary analysis of a randomised controlled trial. Lancet. 2010;375(9721):1173-81. doi:10.1016/S0140-6736(09)62100-0

30. Georgianos PI, Agarwal R. Pharmacotherapy of Hypertension in Chronic Dialysis Patients. Clin J Am Soc Nephrol. 2016;11(11):2062-75. doi:10.2215/CJN.00870116

31. Judd E, Calhoun DA. Management of hypertension in CKD: beyond the guidelines. Adv Chronic Kidney Dis. 2015;22(2):116-22. doi:10.1053/j. ackd.2014.12.001

32. Ku E, Lee BJ, Wei J, Weir MR. Hypertension in CKD: Core Curriculum 2019. Am J Kidney Dis. 2019;74(1):120-31. doi: 10.1053/j.ajkd.2018.12.044..

33. Böhm M, Kario K, Kandzari DE, et al.; SPYRAL HTN-OFF MED Pivotal Investigators. Efficacy of catheter-based renal denervation in the absence of antihypertensive medications (SPYRAL HTN-OFF MED Pivotal): a multicentre, randomised, sham-controlled trial. Lancet. 2020 May 2;395(10234):1444-51. doi: 10.1016/S0140-6736(20)30554-7. 